\title{
Nonlocal Controllability for the Semilinear Fuzzy Integrodifferential Equations in $n$-Dimensional Fuzzy Vector Space
}

\author{
Young Chel Kwun, ${ }^{1}$ Jeong Soon Kim, ${ }^{1}$ Min Ji Park, ${ }^{1}$ \\ and Jin Han Park ${ }^{2}$ \\ ${ }^{1}$ Department of Mathematics, Dong-A University, Pusan 604-714, South Korea \\ ${ }^{2}$ Division of Mathematics Sciences, Pukyong National University, Pusan 608-737, South Korea \\ Correspondence should be addressed to Jin Han Park, jihpark@pknu.ac.kr
}

Received 23 February 2009; Revised 20 June 2009; Accepted 3 August 2009

Recommended by Tocka Diagana

We study the existence and uniqueness of solutions and controllability for the semilinear fuzzy integrodifferential equations in $n$-dimensional fuzzy vector space $\left(E_{N}\right)^{n}$ by using Banach fixed point theorem, that is, an extension of the result of J. H. Park et al. to $n$-dimensional fuzzy vector space.

Copyright (c) 2009 Young Chel Kwun et al. This is an open access article distributed under the Creative Commons Attribution License, which permits unrestricted use, distribution, and reproduction in any medium, provided the original work is properly cited.

\section{Introduction}

Many authors have studied several concepts of fuzzy systems. Diamond and Kloeden [1] proved the fuzzy optimal control for the following system:

$$
\dot{x}(t)=a(t) x(t)+u(t), \quad x(0)=x_{0},
$$

where $x(\cdot)$ and $u(\cdot)$ are nonempty compact interval-valued functions on $E^{1}$. Kwun and Park [2] proved the existence of fuzzy optimal control for the nonlinear fuzzy differential system with nonlocal initial condition in $E_{N}^{1}$ by using Kuhn-Tucker theorems. Fuzzy integrodifferential equations are a field of interest, due to their applicability to the analysis of phenomena with memory where imprecision is inherent. Balasubramaniam and Muralisankar [3] proved the existence and uniqueness of fuzzy solutions for the semilinear fuzzy integrodifferential equation with nonlocal initial condition. They considered the semilinear one-dimensional heat equation on a connected domain $(0,1)$ for material with 
memory. In one-dimensional fuzzy vector space $E_{N^{\prime}}^{1}$ Park et al. [4] proved the existence and uniqueness of fuzzy solutions and presented the sufficient condition of nonlocal controllability for the following semilinear fuzzy integrodifferential equation with nonlocal initial condition:

$$
\begin{gathered}
\frac{d x(t)}{d t}=A\left[x(t)+\int_{0}^{t} G(t-s) x(s) d s\right]+f(t, x)+u(t), \quad t \in J=[0, T] \\
x(0)+g\left(t_{1}, t_{2}, \ldots, t_{p}, x\left(t_{m}\right)\right)=x_{0} \in E_{N}, \quad m=1,2, \ldots, p
\end{gathered}
$$

where $T>0, A: J \rightarrow E_{N}$ is a fuzzy coefficient, $E_{N}$ is the set of all upper semicontinuous convex normal fuzzy numbers with bounded $\alpha$-level intervals, $f: J \times E_{N} \rightarrow E_{N}$ is a nonlinear continuous function, $g: J^{p} \times E_{N} \rightarrow E_{N}$ is a nonlinear continuous function, $G(t)$ is an $n \times n$ continuous matrix such that $d G(t) x / d t$ is continuous for $x \in E_{N}$ and $t \in J$ with $\|G(t)\| \leq K$, $K>0$, with all nonnegative elements, $u: J \rightarrow E_{N}$ is control function.

In [5], Kwun et al. proved the existence and uniqueness of fuzzy solutions for the semilinear fuzzy integrodifferential equations by using successive iteration. In [6], Kwun et al. investigated the continuously initial observability for the semilinear fuzzy integrodifferential equations. Bede and Gal [7] studied almost periodic fuzzy-number-valued functions. Gal and N'Guérékata [8] studied almost automorphic fuzzy-number-valued functions.

In this paper, we study the the existence and uniqueness of solutions and controllability for the following semilinear fuzzy integrodifferential equations:

$$
\begin{gathered}
\frac{d x_{i}(t)}{d t}=A_{i}\left[x_{i}(t)+\int_{0}^{t} G(t-s) x_{i}(s) d s\right]+f_{i}\left(t, x_{i}(t)\right)+u_{i}(t) \text { on } E_{N^{\prime}}^{i} \\
x_{i}(0)+g_{i}\left(x_{i}\right)=x_{0_{i}} \in E_{N}^{i} \quad(i=1,2, \ldots, n),
\end{gathered}
$$

where $A_{i}:[0, T] \rightarrow E_{N}^{i}$ is fuzzy coefficient, $E_{N}^{i}$ is the set of all upper semicontinuously convex fuzzy numbers on $R$ with $E_{N}^{i} \neq E_{N}^{j}(i \neq j), f_{i}:[0, T] \times E_{N}^{i} \rightarrow E_{N}^{i}$ is a nonlinear regular fuzzy function, $g_{i}: E_{N}^{i} \rightarrow E_{N}^{i}$ is a nonlinear continuous function, $G(t)$ is $n \times n$ continuous matrix such that $d G(t) x_{i} / d t$ is continuous for $x_{i} \in E_{N}^{i}$ and $t \in[0, T]$ with $\|G(t)\| \leq k, k>0$, $u_{i}:[0, T] \rightarrow E_{N}^{i}$ is control function and $x_{0_{i}} \in E_{N}^{i}$ is initial value.

\section{Preliminaries}

A fuzzy set of $R^{n}$ is a function $u: R^{n} \rightarrow[0,1]$. For each fuzzy set $u$, we denote by $[u]^{\alpha}=\{x \in$ $\left.R^{n}: u(x) \geq \alpha\right\}$ for any $\alpha \in[0,1]$, its $\alpha$-level set.

Let $u, v$ be fuzzy sets of $R^{n}$. It is well known that $[u]^{\alpha}=[v]^{\alpha}$ for each $\alpha \in[0,1]$ implies $u=v$. conditions:

Let $E^{n}$ denote the collection of all fuzzy sets of $R^{n}$ that satisfies the following

(1) $u$ is normal, that is, there exists an $x_{0} \in R^{n}$ such that $u\left(x_{o}\right)=1$;

(2) $u$ is fuzzy convex, that is, $u(\lambda x+(1-\lambda) y) \geq \min \{u(x), u(y)\}$ for any $x, y \in R^{n}$, $0 \leq \lambda \leq 1$ 
(3) $u(x)$ is upper semicontinuous, that is, $u\left(x_{0}\right) \geq \varlimsup_{\lim _{k \rightarrow \infty}} u\left(x_{k}\right)$ for any $x_{k} \in R^{n}(k=$ $0,1,2, \ldots), x_{k} \rightarrow x_{0}$

(4) $[u]^{0}$ is compact.

We call $u \in E^{n}$ an $n$-dimension fuzzy number.

Wang et al. [9] defined $n$-dimensional fuzzy vector space and investigated its properties.

For any $u_{i} \in E, i=1,2, \ldots, n$, we call the ordered one-dimension fuzzy number class $u_{1}, u_{2}, \ldots, u_{n}$ (i.e., the Cartesian product of one-dimension fuzzy number $u_{1}, u_{2}, \ldots, u_{n}$ ) an $n$ dimension fuzzy vector, denote it as $\left(u_{1}, u_{2}, \ldots, u_{n}\right)$, and call the collection of all $n$-dimension fuzzy vectors (i.e., the Cartesian product $\overbrace{E \times E \times \cdots \times E}$ ) $n$-dimensional fuzzy vector space, and denote it as $(E)^{n}$.

Definition 2.1 (see [9]). If $u \in E^{n}$, and $[u]^{\alpha}$ is a hyperrectangle, that is, $[u]^{\alpha}$ can be represented by $\prod_{i=1}^{n}\left[u_{i l}^{\alpha}, u_{i r}^{\alpha}\right]$, that is, $\left[u_{1 l}^{\alpha}, u_{1 r}^{\alpha}\right] \times\left[u_{2 l}^{\alpha}, u_{2 r}^{\alpha}\right] \times \cdots \times\left[u_{n l}^{\alpha}, u_{n r}^{\alpha}\right]$ for every $\alpha \in[0,1]$, where $u_{i l}^{\alpha}, u_{i r}^{\alpha} \in R$ with $u_{i l}^{\alpha} \leq u_{i r}^{\alpha}$ when $\alpha \in(0,1], i=1,2, \ldots, n$, then we call $u$ a fuzzy $n$-cell number. We denote the collection of all fuzzy $n$-cell numbers by $L\left(E^{n}\right)$.

Theorem 2.2 (see [9]). For any $u \in L\left(E^{n}\right)$ with $[u]^{\alpha}=\prod_{i=1}^{n}\left[u_{i l}^{\alpha}, u_{i r}^{\alpha}\right](\alpha \in[0,1])$, there exists a unique $\left(u_{1}, u_{2}, \ldots, u_{n}\right) \in(E)^{n}$ such that $\left[u_{i}\right]^{\alpha}=\left[u_{i l}^{\alpha}, u_{i r}^{\alpha}\right](i=1,2, \ldots, n$ and $\alpha \in[0,1])$.

Conversely, for any $\left(u_{1}, u_{2}, \ldots, u_{n}\right) \in(E)^{n}$ with $\left[u_{i}\right]^{\alpha}=\left[u_{i l}^{\alpha}, u_{i r}^{\alpha}\right](i=1,2, \ldots, n$ and $\alpha \in$ $[0,1])$, there exists a unique $u \in L\left(E^{n}\right)$ such that $[u]^{\alpha}=\prod_{i=1}^{n}\left[u_{i l}^{\alpha}, u_{i r}^{\alpha}\right](\alpha \in[0,1])$.

Note 1 (see [9]). Theorem 2.2 indicates that fuzzy $n$-cell numbers and $n$-dimension fuzzy vectors can represent each other, so $L\left(E^{n}\right)$ and $(E)^{n}$ may be regarded as identity. If $\left(u_{1}, u_{2}, \ldots, u_{n}\right) \in(E)^{n}$ is the unique $n$-dimension fuzzy vector determined by $u \in L\left(E^{n}\right)$, then we denote $u=\left(u_{1}, u_{2}, \ldots, u_{n}\right)$. $(E)^{n}$.

Let $\left(E_{N}^{i}\right)^{n}=E_{N}^{1} \times E_{N}^{2} \times \cdots \times E_{N^{\prime}}^{n} E_{N}^{i}(i=1,2, \times, n)$ be fuzzy subset of $R$. Then $\left(E_{N}^{i}\right)^{n} \subseteq$

Definition 2.3 (see [9]). The complete metric $D_{L}$ on $\left(E_{N}^{i}\right)^{n}$ is defined by

$$
\begin{aligned}
D_{L}(u, v) & =\sup _{0<\alpha \leq 1} d_{L}\left([u]^{\alpha},[v]^{\alpha}\right) \\
& =\sup _{0<\alpha \leq 1} \max \left\{\left|u_{i l}^{\alpha}-v_{i l}^{\alpha}\right|,\left|u_{i r}^{\alpha}-v_{i r}^{\alpha}\right|\right\}
\end{aligned}
$$

for any $u, v \in\left(E_{N}^{i}\right)^{n}$, which satisfies $d_{L}(u+w, v+w)=d_{L}(u, v)$.

Definition 2.4. Let $u, v \in C\left([0, T]:\left(E_{N}^{i}\right)^{n}\right)$, then

$$
H_{1}(u, v)=\sup _{0 \leq t \leq T} D_{L}(u(t), v(t))
$$


Definition 2.5 (see [9]). The derivative $x^{\prime}(t)$ of a fuzzy process $x \in\left(E_{N}^{i}\right)^{n}$ is defined by

$$
\left[x^{\prime}(t)\right]^{\alpha}=\prod_{i=1}^{n}\left[\left(x_{i l}^{\alpha}\right)^{\prime}(t),\left(x_{i r}^{\alpha}\right)^{\prime}(t)\right]
$$

provided that the equation defines a fuzzy $x^{\prime}(t) \in\left(E_{N}^{i}\right)^{n}$.

Definition 2.6 (see [9]). The fuzzy integral $\int_{b}^{a} x(t) d t, a, b \in[0, \mathrm{~T}]$ is defined by

$$
\left[\int_{b}^{a} x(t) d t\right]^{\alpha}=\prod_{i=1}^{n}\left[\int_{b}^{a} x_{i l}^{\alpha}(t) d t, \int_{b}^{a} x_{i r}^{\alpha}(t) d t\right]
$$

provided that the Lebesgue integrals on the right-hand side exist.

\section{Existence and Uniqueness}

In this section we consider the existence and uniqueness of the fuzzy solution for (1.3) $(u \equiv 0)$. We define

$$
\begin{aligned}
A & =\left(A_{1}, A_{2}, \ldots, A_{n}\right), \\
x & =\left(x_{1}, x_{2}, \ldots, x_{n}\right), \\
f & =\left(f_{1}, f_{2}, \ldots, f_{n}\right), \\
u & =\left(u_{1}, u_{2}, \ldots, u_{n}\right), \\
g & =\left(g_{1}, g_{2}, \ldots, g_{n}\right), \\
x_{0} & =\left(x_{0_{1}}, x_{0_{2}}, \ldots, x_{0_{n}}\right) .
\end{aligned}
$$

Then

$$
A, x, f, x_{0}, u, g \in\left(E_{N}^{i}\right)^{n}
$$
$\left(E_{N}^{i}\right)^{n}:$

Instead of (1.3), we consider the following fuzzy integrodifferential equations in

$$
\begin{gathered}
\frac{d x(t)}{d t}=A\left[x(t)+\int_{0}^{t} G(t-s) x(s) d s\right]+f(t, x(t))+u(t) \text { on }\left(E_{N}^{i}\right)^{n} \\
x(0)+g(x)=x_{0} \in\left(E_{N}^{i}\right)^{n}
\end{gathered}
$$

with fuzzy coefficient $A:[0, T] \rightarrow\left(E_{N}^{i}\right)^{n}$, initial value $x_{0} \in\left(E_{N}^{i}\right)^{n}$, and $u:[0, T] \rightarrow\left(E_{N}^{i}\right)^{n}$ is a control function. Given nonlinear regular fuzzy function $f:[0, T] \times\left(E_{N}^{i}\right)^{n} \rightarrow\left(E_{N}^{i}\right)^{n}$ satisfies a global Lipschitz condition, that is, there exists a finite $k>0$ such that

$$
d_{L}\left([f(s, x(s))]^{\alpha},[f(s, y(s))]^{\alpha}\right) \leq k d_{L}\left([x(s)]^{\alpha},[y(s)]^{\alpha}\right)
$$


for all $x(s), y(s) \in\left(E_{N}^{i}\right)^{n}$. The nonlinear function $g:\left(E_{N}^{i}\right)^{n} \rightarrow\left(E_{N}^{i}\right)^{n}$ is a continuous function and satisfies the Lipschitz condition

$$
d_{L}\left([g(x(\cdot))]^{\alpha},[g(y(\cdot))]^{\alpha}\right) \leq h d_{L}\left([x(\cdot)]^{\alpha},[y(\cdot)]^{\alpha}\right)
$$

for all $x(\cdot), y(\cdot) \in\left(E_{N}^{i}\right)^{n}, h$ is a finite positive constant.

Definition 3.1. The fuzzy process $x: I=[0, T] \rightarrow\left(E_{N}^{i}\right)^{n}$ with $\alpha$-level set $[x(t)]^{\alpha}=\prod_{i=1}^{n}\left[x_{i}\right]^{\alpha}=$ $\Pi_{i=1}^{n}\left[x_{i l}^{\alpha}, x_{i r}^{\alpha}\right]$ is a fuzzy solution of (3.3) without nonhomogeneous term if and only if

$$
\begin{aligned}
& \left(x_{i l}^{\alpha}\right)^{\prime}(t)=\min \left\{A_{i j}^{\alpha}(t)\left[x_{i k}^{\alpha}(t)+\int_{0}^{t} G(t-s) x_{i k}^{\alpha}(s) d s\right]: j, k=l, r\right\}, \\
& \left(x_{i r}^{\alpha}\right)^{\prime}(t)=\max \left\{A_{i j}^{\alpha}(t)\left[x_{i k}^{\alpha}(t)+\int_{0}^{t} G(t-s) x_{i k}^{\alpha}(s) d s\right]: j, k=l, r\right\}, \\
& x_{i l}^{\alpha}(0)+g_{i l}^{\alpha}\left(x_{i l}^{\alpha}\right)=x_{0_{i l},}^{\alpha} \quad x_{i r}^{\alpha}(0)+g_{i r}^{\alpha}\left(x_{i r}^{\alpha}\right)=x_{0 i r}^{\alpha}, \quad i=1,2, \ldots, n .
\end{aligned}
$$

For the sequel, we need the following assumptions.

(H1) $S(t)$ is a fuzzy number satisfying, for $y \in\left(E_{N}^{i}\right)^{n},(d / d t) S(t) y \in C^{1}\left(I:\left(E_{N}^{i}\right)^{n}\right) \cap$ $C\left(I:\left(E_{N}^{i}\right)^{n}\right)$, the equation

$$
\begin{aligned}
\frac{d}{d t} S(t) y & =A\left[S(t) y+\int_{0}^{t} G(t-s) S(s) y d s\right] \\
& =S(t) A y+\int_{0}^{t} S(t-s) A G(s) y d s, \quad t \in I,
\end{aligned}
$$

where

$$
[S(t)]^{\alpha}=\prod_{i=1}^{n}\left[S_{i}(t)\right]^{\alpha}=\prod_{i=1}^{n}\left[S_{i l}^{\alpha}(t), S_{i r}^{\alpha}(t)\right]
$$

and $S_{i j}^{\alpha}(t)(j=l, r)$ is continuous with $\left|S_{i j}^{\alpha}(t)\right| \leq c, c>0$, for all $t \in I=[0, T]$.

(H2) $c\{h(1+T+c T)+k T(1+c T)\}<1$.

In view of Definition 3.1 and (H1), (3.3) can be expressed as

$$
\begin{gathered}
x(t)=S(t)\left(x_{0}-g(x)\right)+\int_{0}^{t} S(t-s)(f(s, x(s))+u(s)) d s, \\
x(0)+g(x)=x_{0} .
\end{gathered}
$$

Theorem 3.2. Let $T>0$. If hypotheses (H1)-(H2) are hold, then for every $x_{0} \in\left(E_{N}^{i}\right)^{n},(3.9)(u \equiv 0)$ have a unique fuzzy solution $x \in C\left([0, T]:\left(E_{N}^{i}\right)^{n}\right)$. 
Proof. For each $x(t) \in\left(E_{N}^{i}\right)^{n}$ and $t \in[0, T]$, define $\left(G_{0} x\right)(t) \in\left(E_{N}^{i}\right)^{n}$ by

$$
\left(G_{0} x\right)(t)=S(t)\left(x_{0}-g(x)\right)+\int_{0}^{t} S(t-s) f(s, x(s)) d s
$$

Thus, $G_{0} x:[0, T] \rightarrow\left(E_{N}^{i}\right)^{n}$ is continuous, so $G_{0}$ is a mapping from $C\left([0, T]:\left(E_{N}^{i}\right)^{n}\right)$ into itself. By Definitions 2.3 and 2.4, some properties of $d_{L}$, and inequalities (3.4) and (3.5), we have following inequalities. For $x, y \in C\left([0, T]:\left(E_{N}^{i}\right)^{n}\right)$,

$$
\begin{aligned}
d_{L}( & {\left.\left[\left(G_{0} x\right)(t)\right]^{\alpha},\left[\left(G_{0} y\right)(t)\right]^{\alpha}\right) } \\
= & d_{L}\left(\left[S(t)\left(x_{0}-g(x)\right)+\int_{0}^{t} S(t-s) f(s, x(s)) d s\right]^{\alpha},\right. \\
& \left.\quad\left[S(t)\left(x_{0}-g(y)\right)+\int_{0}^{t} S(t-s) f(s, y(s)) d s\right]^{\alpha}\right) \\
= & d_{L}\left(\left[-S(t) g(x)+\int_{0}^{t} S(t-s) f(s, x(s)) d s\right]^{\alpha},\right. \\
& \left.\quad\left[-S(t) g(y)+\int_{0}^{t} S(t-s) f(s, y(s)) d s\right]^{\alpha}\right) \\
\leq & d_{L}\left([S(t) g(x)]^{\alpha},[S(t) g(y)]^{\alpha}\right)+\int_{0}^{t} d_{L}\left([S(t-s) f(s, x(s))]^{\alpha},[S(t-s) f(s, y(s))]^{\alpha}\right) d s \\
= & \max _{1 \leq i \leq n}\left\{\left|S_{i l}^{\alpha}(t)\left(g_{i l}^{\alpha}(x)-g_{i l}^{\alpha}(y)\right)\right|,\left|S_{i r}^{\alpha}(t)\left(g_{i r}^{\alpha}(x)-g_{i r}^{\alpha}(y)\right)\right|\right\} \\
\leq & c h d_{L}\left([x(\cdot)]^{\alpha},[y(\cdot)]^{\alpha}\right)+c k \int_{0}^{t} d_{L}\left([x(s)]^{\alpha},[y(s)]^{\alpha}\right) d s . \\
+ & \int_{0}^{t} \max _{1 \leq i \leq n}\left\{\left|S_{i l}^{\alpha}(t-s)\left(f_{i l}^{\alpha}(s, x(s))-f_{i l}^{\alpha}(s, y(s))\right)\right|,\left|S_{i r}^{\alpha}(t-s)\left(f_{i r}^{\alpha}(s, x(s))-f_{i r}^{\alpha}(s, y(s))\right)\right|\right\} d s \\
\leq & c \max _{1 \leq i \leq n}\left\{\left|\left(g_{i l}^{\alpha}(x)-g_{i l}^{\alpha}(y)\right)\right|,\left|\left(g_{i r}^{\alpha}(x)-g_{i r}^{\alpha}(y)\right)\right|\right\} \\
+ & c \int_{0}^{t} \max _{1 \leq i \leq n}\left\{\left|f_{i l}^{\alpha}(s, x(s))-f_{i l}^{\alpha}(s, y(s))\right|,\left|f_{i r}^{\alpha}(s, x(s))-f_{i r}^{\alpha}(s, y(s))\right|\right\} d s \\
= &
\end{aligned}
$$


Therefore

$$
\begin{aligned}
& D_{L}\left(\left(G_{0} x\right)(t),\left(G_{0} y\right)(t)\right) \\
& \quad=\sup _{0<\alpha \leq 1} d_{L}\left(\left[\left(G_{0} x\right)(t)\right]^{\alpha},\left[\left(G_{0} y\right)(t)\right]^{\alpha}\right) \\
& \quad \leq \operatorname{chsup} d_{0<\alpha \leq 1}\left([x(\cdot)]^{\alpha},[y(\cdot)]^{\alpha}\right)+c k \sup _{0<\alpha \leq 1} \int_{0}^{t} d_{L}\left([x(s)]^{\alpha},[y(s)]^{\alpha}\right) d s \\
& \quad \leq \operatorname{ch} D_{L}(x(\cdot), y(\cdot))+c k \int_{0}^{t} D_{L}(x(s), y(s)) d s .
\end{aligned}
$$

Hence

$$
\begin{aligned}
H_{1}\left(G_{0} x, G_{0} y\right) & =\sup _{0 \leq t \leq T} D_{L}\left(\left(G_{0} x\right)(t),\left(G_{0} y\right)(t)\right) \\
& \leq \operatorname{chsup} D_{0 \leq t \leq T} D_{L}(x(\cdot), y(\cdot))+c k \sup _{0 \leq t \leq T} \int_{0}^{t} D_{L}(x(s), y(s)) d s \\
& \leq c h H_{1}(x, y)+c k T H_{1}(x, y) \\
& =c(h+k T) H_{1}(x, y) .
\end{aligned}
$$

By hypothesis $(\mathrm{H} 2), \mathrm{G}_{0}$ is a contraction mapping. $\left.\left(E_{N}^{i}\right)^{n}\right)$.

Using the Banach fixed point theorem, (3.9) have a unique fixed point $x \in C([0, T]$ :

\section{Controllability}

In this section, we show the nonlocal controllability for the control system (1.3).

Definition 4.1. Equation (1.3) is nonlocal controllable. Then there exists $u(t)$ such that the fuzzy solution $x(t)$ for (3.9) as $x(T)=x^{1}-g(x)$ (i.e., $[x(T)]^{\alpha}=\left[x^{1}-g(x)\right]^{\alpha}$ ) where $x^{1} \in\left(E_{N}^{i}\right)^{n}$ is target set.

Define the fuzzy mapping $\tilde{\beta}: \widetilde{P}\left(R^{n}\right) \rightarrow\left(E_{N}^{i}\right)^{n}$ by

$$
\tilde{\beta}^{\alpha}(v)= \begin{cases}\int_{0}^{T} S^{\alpha}(T-s) v(s) d s, & v \subset \bar{\Gamma}_{u} \\ 0, & \text { otherwise }\end{cases}
$$


where $\bar{\Gamma}_{u}$ is closed support of $u$. Then there exists

$$
\tilde{\beta}_{i}: \tilde{P}(R) \longrightarrow E_{N}^{i} \quad(i=1,2, \ldots, n)
$$

such that

$$
\tilde{\beta}_{i}^{\alpha}\left(v_{i}\right)= \begin{cases}\int_{0}^{T} S_{i}^{\alpha}(T-s) v_{i}(s) d s, & v_{i}(s) \subset \bar{\Gamma}_{u_{i}}, \\ 0, & \text { otherwise. }\end{cases}
$$

Then $\tilde{\beta}_{i j}^{\alpha}(j=l, r)$ exists such that

$$
\begin{array}{ll}
\tilde{\beta}_{i l}^{\alpha}\left(v_{i l}\right)=\int_{0}^{T} S_{i l}^{\alpha}(T-s) v_{i l}(s) d s, & v_{i l}(s) \in\left[u_{i l}^{\alpha}(s), u_{i}^{1}\right] \\
\tilde{\beta}_{i r}^{\alpha}\left(v_{i r}\right)=\int_{0}^{T} S_{i r}^{\alpha}(T-s) v_{i r}(s) d s, & v_{i r}(s) \in\left[u_{i}^{1}, u_{i r}^{\alpha}(s)\right] .
\end{array}
$$

We assume that $\tilde{\beta}_{i l}^{\alpha}, \tilde{\beta}_{i r}^{\alpha}$ are bijective mappings.

We can introduce $\alpha$-level set of $u(s)$ of (3.4)-(3.5)

$$
\begin{aligned}
& {[u(s)]^{\alpha}=} \prod_{i=1}^{n}\left[u_{i}(s)\right]^{\alpha} \\
&= \prod_{i=1}^{n}\left[u_{i l}^{\alpha}(s), u_{i r}^{\alpha}(s)\right] \\
&= \prod_{i=1}^{n}\left[( \tilde { \beta } _ { i l } ^ { \alpha } ) ^ { - 1 } \left(\left(\left(x^{1}\right)_{i l}^{\alpha}-g_{i l}^{\alpha}\left(x_{i l}^{\alpha}\right)\right)-S_{i l}^{\alpha}(T)\left(x_{0_{i l}}^{\alpha}-g_{i l}^{\alpha}\left(x_{i l}^{\alpha}\right)\right)\right.\right. \\
&\left.\quad-\int_{0}^{T} S_{i l}^{\alpha}(T-s) f_{i l}^{\alpha}\left(s, x_{i l}^{\alpha}(s)\right) d s\right), \\
&\left(\tilde{\beta}_{i r}^{\alpha}\right)^{-1}\left(\left(x^{1}\right)_{i r}^{\alpha}-g_{i r}^{\alpha}\left(x_{i r}^{\alpha}\right)\right)-S_{i r}^{\alpha}(T)\left(x_{0_{i r}}^{\alpha}-g_{i r}^{\alpha}\left(x_{i r}^{\alpha}\right)\right) \\
&\left.\left.\quad-\int_{0}^{T} S_{i r}^{\alpha}(T-s) f_{i r}^{\alpha}\left(s, x_{i r}^{\alpha}(s)\right) d s\right)\right] .
\end{aligned}
$$

Then substituting this expression into (3.9) yields $\alpha$-level of $x(T)$. 
For each $i=1,2, \ldots, n$,

$$
\begin{aligned}
& {\left[x_{i}(T)\right]^{\alpha}=[} S_{i l}^{\alpha}(T)\left(x_{0_{i l}}^{\alpha}-g_{i l}^{\alpha}\left(x_{i l}^{\alpha}\right)\right)+\int_{0}^{T} S_{i l}^{\alpha}(T-s) f_{i l}^{\alpha}\left(s, x_{i l}^{\alpha}(s)\right) d s \\
&+\int_{0}^{T} S_{i l}^{\alpha}(T-s)\left(\tilde{\beta}_{i l}^{\alpha}\right)^{-1}\left(\left(x^{1}\right)_{i l}^{\alpha}-g_{i l}^{\alpha}\left(x_{i l}^{\alpha}\right)\right)-S_{i l}^{\alpha}(T)\left(x_{0_{i l}}^{\alpha}-g_{i l}^{\alpha}\left(x_{i l}^{\alpha}\right)\right) \\
&\left.\quad-\int_{0}^{T} S_{i l}^{\alpha}(T-s) f_{i l}^{\alpha}\left(s, x_{i l}^{\alpha}(s)\right) d s\right) d s, \\
& S_{i r}^{\alpha}(T)\left(x_{0_{i r}}^{\alpha}-g_{i r}^{\alpha}\left(x_{i r}^{\alpha}\right)\right)+\int_{0}^{T} S_{i r}^{\alpha}(T-s) f_{i r}^{\alpha}\left(s, x_{i r}^{\alpha}(s)\right) d s \\
&+\int_{0}^{T} S_{i r}^{\alpha}(T-s)\left(\tilde{\beta}_{i r}^{\alpha}\right)^{-1}\left(\left(\left(x^{1}\right)_{i r}^{\alpha}-g_{i r}^{\alpha}\left(x_{i r}^{\alpha}\right)\right)-S_{i r}^{\alpha}(T)\left(x_{0 i r}^{\alpha}-g_{i r}^{\alpha}\left(x_{i r}^{\alpha}\right)\right)\right. \\
&\left.\left.-\int_{0}^{T} S_{i r}^{\alpha}(T-s) f_{i r}^{\alpha}\left(s, x_{i r}^{\alpha}(s)\right) d s\right) d s\right] \\
&=\left[\left(x^{1}-g(x)\right)_{i l^{\prime}}^{\alpha}\left(x^{1}-g(x)\right)_{i r}^{\alpha}\right]=\left[\left(x^{1}-g(x)\right)_{i}\right]^{\alpha} .
\end{aligned}
$$

Therefore

$$
[x(T)]^{\alpha}=\prod_{i=1}^{n}\left[x_{i}(T)\right]^{\alpha}=\prod_{i=1}^{n}\left[\left(x^{1}-g(x)\right)_{i}\right]^{\alpha}=\left[x^{1}-g(x)\right]^{\alpha} .
$$

We now set

$$
\begin{aligned}
\Phi x(t)= & S(t)\left(x_{0}-g(x)\right)+\int_{0}^{t} S(t-s) f(s, x(s)) d s \\
& +\int_{0}^{t} S(t-s) \tilde{\beta}^{-1}\left(x^{1}-g(x)-S(T)\left(x_{0}-g(x)\right)-\int_{0}^{T} S(T-s) f(s, x(s)) d s\right) d s,
\end{aligned}
$$

where the fuzzy mapping $\widetilde{\beta}^{-1}$ satisfies above statements.

Notice that $\Phi x(T)=x^{1}-g(x)$, which means that the control $u(t)$ steers (3.9) from the origin to $x^{1}-g(x)$ in time $T$ provided that we can obtain a fixed point of the operator $\Phi$.

(H3) Assume that the linear system of (3.9) $(f \equiv 0)$ is controllable.

Theorem 4.2. Suppose that hypotheses (H1)-(H3) are satisfied. Then (3.9) are nonlocal controllable. 
Proof. We can easily check that $\Phi$ is continuous function from $C\left([0, T]:\left(E_{N}^{i}\right)^{n}\right)$ to itself. By Definitions 2.3 and 2.4, some properties of $d_{L}$, and inequalities (3.4) and (3.5), we have the following inequalities. For any $x, y \in C\left([0, T]:\left(E_{N}^{i}\right)^{n}\right)$,

$$
\begin{aligned}
& d_{L}\left([\Phi x(t)]^{\alpha},[\Phi y(t)]^{\alpha}\right) \\
& =d_{L}\left(\left[S(t)\left(x_{0}-g(x)\right)+\int_{0}^{t} S(t-s) f(s, x(s)) d s+\int_{0}^{t} S(t-s) \tilde{\beta}^{-1}\right.\right. \\
& \left.\times\left(x^{1}-g(x)-S(T)\left(x_{0}-g(x)\right)-\int_{0}^{T} S(T-s) f(s, x(s)) d s\right) d s\right]^{\alpha}, \\
& {\left[S(t)\left(x_{0}-g(y)\right)+\int_{0}^{t} S(t-s) f(s, y(s)) d s+\int_{0}^{t} S(t-s) \tilde{\beta}^{-1}\right.} \\
& \left.\left.\times\left(x^{1}-g(y)-S(T)\left(x_{0}-g(y)\right)-\int_{0}^{T} S(T-s) f(s, y(s)) d s\right) d s\right]^{\alpha}\right) \\
& \leq d_{L}\left([S(t) g(x)]^{\alpha},[S(t) g(y)]^{\alpha}\right)+\int_{0}^{t} d_{L}\left([S(t-s) f(s, x(s))]^{\alpha},[S(t-s) f(s, y(s))]^{\alpha}\right) d s \\
& +\int_{0}^{t} d_{L}\left(\left[S(t-s) \tilde{\beta}^{-1} g(x)\right]^{\alpha},\left[S(t-s) \tilde{\beta}^{-1} g(y)\right]^{\alpha}\right) d s \\
& +\int_{0}^{t} d_{L}\left(\left[S(t-s) \tilde{\beta}^{-1} S(T) g(x)\right]^{\alpha},\left[S(t-s) \tilde{\beta}^{-1} S(T) g(y)\right]^{\alpha}\right) d s \\
& +\int_{0}^{t} d_{L}\left(\left[S(t-s) \tilde{\beta}^{-1} \int_{0}^{T} S(T-s) f(s, x(s)) d s\right]^{\alpha},\left[S(t-s) \tilde{\beta}^{-1} \int_{0}^{T} S(T-s) f(s, y(s)) d s\right]^{\alpha}\right) d s \\
& =\max _{1 \leq i \leq n}\left\{\left|S_{i l}^{\alpha}(t)\left(g_{i l}^{\alpha}(x)-g_{i l}^{\alpha}(y)\right)\right|,\left|S_{i r}^{\alpha}(t)\left(g_{i r}^{\alpha}(x)-g_{i r}^{\alpha}(y)\right)\right|\right\} \\
& +\int_{0}^{t} \max _{0 \leq i \leq n}\left\{\left|S_{i l}^{\alpha}(t-s)\left(f_{i l}^{\alpha}(s, x(s))-f_{i l}^{\alpha}(s, y(s))\right)\right|,\left|S_{i r}^{\alpha}(t-s)\left(f_{i r}^{\alpha}(s, x(s))-f_{i r}^{\alpha}(s, y(s))\right)\right|\right\} d s \\
& +\int_{0}^{t} \max _{0 \leq i \leq n}\left\{\left|S_{i l}^{\alpha}(t-s)\left(\tilde{\beta}_{i l}^{\alpha}\right)^{-1}\left(g_{i l}^{\alpha}(x)-g_{i l}^{\alpha}(y)\right)\right|,\left|S_{i r}^{\alpha}(t-s)\left(\tilde{\beta}_{i r}^{\alpha}\right)^{-1}\left(g_{i r}^{\alpha}(x)-g_{i r}^{\alpha}(y)\right)\right|\right\} d s \\
& +\int_{0}^{t} \max _{1 \leq i \leq n}\left\{\left|S_{i l}^{\alpha}(t-s)\left(\tilde{\beta}_{i l}^{\alpha}\right)^{-1} S_{i l}^{\alpha}(T)\left(g_{i l}^{\alpha}(x)-g_{i l}^{\alpha}(y)\right)\right|,\right. \\
& \left.\left|S_{i r}^{\alpha}(t-s)\left(\tilde{\beta}_{i r}^{\alpha}\right)^{-1} S_{i r}^{\alpha}(T)\left(g_{i r}^{\alpha}(x)-g_{i r}^{\alpha}(y)\right)\right|\right\} d s \\
& +\int_{0}^{t} \max _{1 \leq i \leq n}\left\{\left|S_{i l}^{\alpha}(t-s)\left(\tilde{\beta}_{i l}^{\alpha}\right)^{-1}\left(\int_{0}^{T} S_{i l}^{\alpha}(T-s) f_{i l}^{\alpha}(s, x(s)) d s-\int_{0}^{T} S_{i l}^{\alpha}(T-s) f_{i l}^{\alpha}(s, y(s)) d s\right)\right|,\right. \\
& \left.\left|S_{i r}^{\alpha}(t-s)\left(\tilde{\beta}_{i l}^{\alpha}\right)^{-1}\left(\int_{0}^{T} S_{i r}^{\alpha}(T-s) f_{i r}^{\alpha}(s, x(s)) d s-\int_{0}^{T} S_{i r}^{\alpha}(T-s) f_{i r}^{\alpha}(s, y(s)) d s\right)\right|\right\} d s
\end{aligned}
$$




$$
\begin{aligned}
\leq & c \max _{1 \leq i \leq n}\left\{\left|g_{i l}^{\alpha}(x)-g_{i l}^{\alpha}(y)\right|,\left|g_{i r}^{\alpha}(x)-g_{i r}^{\alpha}(y)\right|\right\} \\
& +c \int_{0}^{t} \max _{1 \leq i \leq n}\left\{\left|f_{i l}^{\alpha}(s, x(s))-f_{i l}^{\alpha}(s, y(s))\right|,\left|f_{i r}^{\alpha}(s, x(s))-f_{i r}^{\alpha}(s, y(s))\right|\right\} d s \\
& +c \int_{0}^{t} \max _{1 \leq i \leq n}\left\{\left|g_{i l}^{\alpha}(x)-g_{i l}^{\alpha}(y)\right|,\left|g_{i r}^{\alpha}(x)-g_{i r}^{\alpha}(y)\right|\right\} d s \\
& +c^{2} \int_{0}^{t} \max _{1 \leq i \leq n}\left\{\left|g_{i l}^{\alpha}(x)-g_{i l}^{\alpha}(y)\right|,\left|g_{i r}^{\alpha}(x)-g_{i r}^{\alpha}(y)\right|\right\} d s \\
& +c^{2} \int_{0}^{t} \int_{0}^{T} \max _{1 \leq i \leq n}\left\{\left|f_{i l}^{\alpha}(s, x(s))-f_{i l}^{\alpha}(s, y(s))\right|,\left|f_{i r}^{\alpha}(s, x(s))-f_{i r}^{\alpha}(s, y(s))\right|\right\} d s d s \\
= & c d_{L}\left([g(x)]^{\alpha},[g(y)]^{\alpha}\right)+c \int_{0}^{t} d_{L}\left([f(s, x(s))]^{\alpha},[f(s, y(s))]^{\alpha}\right) d s \\
& +c \int_{0}^{t} d_{L}\left([g(x)]^{\alpha},[g(y)]^{\alpha}\right) d s+c^{2} \int_{0}^{t} d_{L}\left([g(x)]^{\alpha},[g(y)]^{\alpha}\right) d s \\
& +c^{2} \int_{0}^{t} \int_{0}^{T} d_{L}\left([f(s, x(s))]^{\alpha},[f(s, y(s))]^{\alpha}\right) d s d s \\
\leq & c h\left\{d_{L}\left([x(\cdot)]^{\alpha},[y(\cdot)]^{\alpha}\right)+(1+c) \int_{0}^{t} d_{L}\left([x(\cdot)]^{\alpha},[y(\cdot)]^{\alpha}\right) d s\right\} \\
& +c k\left\{\int_{0}^{t} d_{L}\left([x(s)]^{\alpha},[y(s)]^{\alpha}\right) d s+c \int_{0}^{t} \int_{0}^{T} d_{L}\left([x(s)]^{\alpha},[y(s)]^{\alpha}\right) d s d s\right\} .
\end{aligned}
$$

Therefore

$$
\begin{aligned}
D_{L}(\Phi x(t), \Phi y(t)) \\
=\sup _{0<\alpha \leq 1} d_{L}\left([\Phi x(t)]^{\alpha},[\Phi y(t)]^{\alpha}\right) \\
\leq \operatorname{ch}\left\{\sup _{0<\alpha \leq 1} d_{L}\left([x(\cdot)]^{\alpha},[y(\cdot)]^{\alpha}\right)+(1+c) \int_{0}^{t} \sup _{0<\alpha \leq 1} d_{L}\left([x(\cdot)]^{\alpha},[y(\cdot)]^{\alpha}\right) d s\right\} \\
\quad+c k\left\{\int_{0}^{t} \sup _{0<\alpha \leq 1} d_{L}\left([x(s)]^{\alpha},[y(s)]^{\alpha}\right) d s+c \int_{0}^{t} \int_{0<\alpha \leq 1}^{T} \sup _{L} d_{L}\left([x(s)]^{\alpha},[y(s)]^{\alpha}\right) d s d s\right\} \\
=\operatorname{ch}\left\{D_{L}(x(\cdot), y(\cdot))+(1+c) \int_{0}^{t} D_{L}(x(\cdot), y(\cdot)) d s\right\} \\
\quad+c k\left\{\int_{0}^{t} D_{L}(x(s), y(s)) d s+c \int_{0}^{t} \int_{0}^{T} D_{L}(x(s), y(s)) d s d s\right\} .
\end{aligned}
$$


Hence

$$
\begin{aligned}
H_{1}(\Phi x, \Phi y)= & \sup _{0 \leq t \leq T} D_{L}(\Phi x(t), \Phi y(t)) \\
\leq & c h\left\{\sup _{0 \leq t \leq T} D_{L}(x(\cdot), y(\cdot))+(1+c) \sup _{0 \leq t \leq T} \int_{0}^{t} D_{L}(x(\cdot), y(\cdot)) d s\right\} \\
& +c k\left\{\sup _{0 \leq t \leq T} \int_{0}^{t} D_{L}(x(s), y(s)) d s+\operatorname{csup}_{0 \leq t \leq T} \int_{0}^{t} \int_{0}^{T} D_{L}(x(s), y(s)) d s d s\right\} \\
\leq & c h\left\{H_{1}(x, y)+(1+c) T H_{1}(x, y)\right\}+c k\left\{T H_{1}(x, y)+c T^{2} H_{1}(x, y)\right\} \\
= & c\{h(1+T+c T)+k T(1+c T)\} H_{1}(x, y) .
\end{aligned}
$$

By hypothesis $(\mathrm{H} 2), \Phi$ is a contraction mapping. Using the Banach fixed point theorem, (4.8) has a unique fixed point $x \in C\left([0, T]:\left(E_{N}^{i}\right)^{n}\right)$.

\section{Example}

Consider the two semilinear one-dimensional heat equations on a connected domain $(0,1)$ for material with memory on $E_{N^{\prime}}^{i} i=1,2$, boundary condition $x_{i}(t, 0)=x_{i}(t, 1)=0$, $i=1,2$ and with initial conditions $x_{i}\left(0, z_{i}\right)+\sum_{k=1}^{p}\left(c_{k}\right)_{i} x_{i}\left(t_{k}, z_{i}\right)=x_{0_{i}}\left(z_{i}\right)$, where $x_{0_{i}}\left(z_{i}\right) \in$ $E_{N}^{i}, \sum_{k=1}^{p}\left(c_{k}\right)_{i} x_{i}\left(t_{k}, z_{i}\right)=g_{i}\left(x_{i}\right), i=1,2$. Let $x_{i}\left(t, z_{i}\right), i=1,2$, be the internal energy and let $f_{i}\left(t, x_{i}\left(t, z_{i}\right)\right)=\tilde{2} t x_{i}\left(t, z_{i}\right)^{2}, i=1,2$, be the external heat.

Let

$$
\begin{gathered}
A=\left(A_{1}, A_{2}\right)=\left(\tilde{2} \frac{\partial^{2}}{\partial z_{1}^{2}}, \tilde{2} \frac{\partial^{2}}{\partial z_{2}^{2}}\right), \\
f(t, x(t))=\left(f_{1}\left(t, x_{1}(t)\right), f_{2}\left(t, x_{2}(t)\right)\right)=\left(\widetilde{2} t x_{1}\left(t, z_{1}\right)^{2}, \widetilde{2} t x_{2}\left(t, z_{2}\right)^{2}\right), \\
g(x)=\left(g_{1}\left(x_{1}\right), g_{2}\left(x_{2}\right)\right)=\left(\sum_{k=1}^{p}\left(c_{k}\right) x_{1}\left(t_{k}, z_{1}\right), \sum_{k=1}^{p}\left(c_{k}\right)_{2} x_{2}\left(t_{k}, z_{2}\right)\right), \\
x(0)+g(x)=\left(x_{1}(0)+g_{1}(x), x_{2}(0)+g_{2}(x)\right), \quad x_{0}=\left(x_{0_{1}}, x_{0_{2}}\right)=(\tilde{0}, \tilde{0}), \\
G(t-s)=\left(e^{-(t-s)}, e^{-(t-s)}\right),
\end{gathered}
$$

then the balance equations become

$$
\begin{gathered}
\frac{d x(t)}{d t}=A\left[x(t)+\int_{0}^{t} G(t-s) x(s) d s\right]+f(t, x(t)) \text { on }\left(E_{N}^{i}\right)^{2}, \\
x(0)+g(x)=x_{0} \in\left(E_{N}^{i}\right)^{2} .
\end{gathered}
$$


The $\alpha$-level sets of fuzzy numbers are the following: [0̃ $]^{\alpha}=[\alpha-1,1-\alpha],[\tilde{2}]^{\alpha}=[\alpha+$ $1,3-\alpha]$ for all $\alpha \in[0,1]$. Then $\alpha$-level set of $f(t, x(t))$ is

$$
\begin{aligned}
& {[f(t, x(t))]^{\alpha}} \\
& =\left[\tilde{2} t x_{1}(t)^{2}\right]^{\alpha} \times\left[\tilde{2} t x_{2}(t)^{2}\right]^{\alpha} \\
& =[\tilde{2}]^{\alpha} \cdot t\left[x_{1}(t)^{2}\right]^{\alpha} \times[\tilde{2}]^{\alpha} \cdot t\left[x_{2}(t)^{2}\right]^{\alpha} \\
& =[\alpha+1,3-\alpha] \cdot t\left[\left(x_{1 l}^{\alpha}(t)\right)^{2},\left(x_{1 r}^{\alpha}(t)\right)^{2}\right] \times[\alpha+1,3-\alpha] \cdot t\left[\left(x_{2 l}^{\alpha}(t)\right)^{2},\left(x_{2 r}^{\alpha}(t)\right)^{2}\right] \\
& =\left[(\alpha+1) t\left(x_{1 l}^{\alpha}(t)\right)^{2},(3-\alpha) t\left(x_{1 r}^{\alpha}(t)\right)^{2}\right] \times\left[(\alpha+1) t\left(x_{2 l}^{\alpha}(t)\right)^{2},(3-\alpha) t\left(x_{2 r}^{\alpha}(t)\right)^{2}\right] .
\end{aligned}
$$

Further, we have

$$
\begin{aligned}
d_{L} & \left.\left([f(t, x(t))]^{\alpha}, f(t, y(t))\right]^{\alpha}\right) \\
& =d_{L}\left(\left[(\alpha+1) t\left(x_{i l}^{\alpha}(t)\right)^{2},(3-\alpha) t\left(x_{i r}^{\alpha}(t)\right)^{2}\right],\left[(\alpha+1) t\left(y_{i l}^{\alpha}(t)\right)^{2},(3-\alpha) t\left(y_{i r}^{\alpha}(t)\right)^{2}\right]\right) \\
& =t \max _{1 \leq i \leq 2}\left\{(\alpha+1)\left|\left(x_{i l}^{\alpha}(t)\right)^{2}-\left(y_{i l}^{\alpha}(t)\right)^{2}\right|,(3-\alpha)\left|\left(x_{i r}^{\alpha}(t)\right)^{2}-\left(y_{i r}^{\alpha}(t)\right)^{2}\right|\right\} \\
& \leq T(3-\alpha) \max _{1 \leq i \leq 2}\left\{\left|x_{i l}^{\alpha}(t)-y_{i l}^{\alpha}(t)\right|\left|x_{i l}^{\alpha}(t)+y_{i l}^{\alpha}(t)\right|,\left|x_{i r}^{\alpha}(t)-y_{i r}^{\alpha}(t)\right|\left|x_{i r}^{\alpha}(t)+y_{i r}^{\alpha}(t)\right|\right\} \\
& \leq 3 T\left|x_{i r}^{\alpha}(t)+y_{i r}^{\alpha}(t)\right| \times \max _{1 \leq i \leq 2}\left\{\left|x_{i l}^{\alpha}(t)-y_{i l}^{\alpha}(t)\right|,\left|x_{i r}^{\alpha}(t)-y_{i r}^{\alpha}(t)\right|\right\} \\
& =k d_{L}\left([x(t)]^{\alpha},[y(t)]^{\alpha}\right), \\
d_{L} & \left([g(x(\cdot))]^{\alpha},[g(y(\cdot))]^{\alpha}\right) \\
& =d_{L}\left(\left[\sum_{k=1}^{p} c_{k}\left(x\left(t_{k}\right)\right)\right]^{\alpha},\left[\sum_{k=1}^{p} c_{k}\left(y\left(t_{k}\right)\right)\right]^{\alpha}\right) \\
& =\max _{1 \leq i \leq 2}\left\{\left|\sum_{k=1}^{p}\left(c_{k}\right)_{i}\left(x_{i l}^{\alpha}\left(t_{k}\right)\right)-\sum_{k=1}^{p}\left(c_{k}\right)_{i}\left(y_{i l}^{\alpha}\left(t_{k}\right)\right)\right|,\left|\sum_{k=1}^{p}\left(c_{k}\right)_{i}\left(x_{i r}^{\alpha}\left(t_{k}\right)\right)-\sum_{k=1}^{p}\left(c_{k}\right)_{i}\left(y_{i r}^{\alpha}\left(t_{k}\right)\right)\right|\right\} \\
& \leq\left|\sum_{k=1}^{p} c_{k}\right| \max _{1 \leq i \leq 2}\left\{\left|x_{i l}^{\alpha}\left(t_{k}\right)-y_{i l}^{\alpha}\left(t_{k}\right)\right|,\left|x_{i r}^{\alpha}\left(t_{k}\right)-y_{i r}^{\alpha}\left(t_{k}\right)\right|\right\} \\
& \leq\left|\sum_{k=1}^{p} c_{k}\right| \max _{k}^{p} d_{L}\left(\left[x\left(t_{k}\right)\right]^{\alpha},\left[y\left(t_{k}\right)\right]^{\alpha}\right) \\
& =h d_{L}\left([x(\cdot)]^{\alpha},[y(\cdot)]^{\alpha}\right), \\
& \left|\sum_{k=1}^{p} c_{k}\right| d_{L}\left(\left[x\left(t_{k}\right)\right]^{\alpha},\left[y\left(t_{k}\right)\right]^{\alpha}\right) \\
&
\end{aligned}
$$


where $k$ and $h$ satisfy the inequality (3.4) and (3.5), respectively. Choose $T$ such that $T<$ $(1-c h) / c k$. Then all conditions stated in Theorem 3.2 are satisfied, so problem (5.2) has a unique fuzzy solution.

Let target set be $x^{1}=\left(x_{1}^{1}, x_{2}^{1}\right)=(\tilde{2}, \tilde{3})$. The $\alpha$-level set of fuzzy numbers is $\tilde{3}[\tilde{3}]^{\alpha}=$ $[\alpha+2,4-\alpha]$.

From the definition of fuzzy solution,

$$
\begin{aligned}
x_{i l}^{\alpha}(t)= & S_{i l}^{\alpha}(t)\left(\left(x_{0}\right)_{i l}^{\alpha}-\sum_{k=1}^{p}\left(c_{k}\right)_{i}\left(x_{i l}^{\alpha}\left(t_{k}\right)\right)\right) \\
& +\int_{0}^{t} S_{i l}^{\alpha}(t-s)(\alpha+1) s\left(x_{i l}^{\alpha}(s)\right)^{2} d s+\int_{0}^{t} S_{i l}^{\alpha}(t-s) u_{i l}^{\alpha}(s) d s, \\
x_{i r}^{\alpha}(t)= & S_{i r}^{\alpha}(t)\left(\left(x_{0}\right)_{i r}^{\alpha}-\sum_{k=1}^{p}\left(c_{k}\right)_{i}\left(x_{i r}^{\alpha}\left(t_{k}\right)\right)\right) \\
& +\int_{0}^{t} S_{i r}^{\alpha}(t-s)(3-\alpha) s\left(x_{i r}^{\alpha}(s)\right)^{2} d s+\int_{0}^{t} S_{i r}^{\alpha}(t-s) u_{i r}^{\alpha}(s) d s,
\end{aligned}
$$

where $i=1,2$.

Thus the $\alpha$-level of $u(s)$ is

$$
\begin{aligned}
u_{1 l}^{\alpha}(s)=\left(\tilde{\beta}_{1 l}^{\alpha}\right)^{-1}( & (\alpha+1)-\sum_{k=1}^{p}\left(c_{k}\right)_{1}\left(x_{i l}^{\alpha}\left(t_{k}\right)\right) \\
& \left.-\left[S_{1 l}^{\alpha}(T)\left(\left(x_{0}\right)_{1 l}^{\alpha}-\sum_{k=1}^{p}\left(c_{k}\right)_{1}\left(x_{1 l}^{\alpha}\left(t_{k}\right)\right)\right)+\int_{0}^{T}(\alpha+1) S_{1 l}^{\alpha}(T-s) s\left(x_{1 l}^{\alpha}(s)\right)^{2} d s\right]\right), \\
u_{1 r}^{\alpha}(s)=\left(\tilde{\beta}_{1 r}^{\alpha}\right)^{-1}( & (3-\alpha)-\sum_{k=1}^{p}\left(c_{k}\right)_{1}\left(x_{i r}^{\alpha}\left(t_{k}\right)\right) \\
- & {\left.\left[S_{1 r}^{\alpha}(T)\left(\left(x_{0}\right)_{1 r}^{\alpha}-\sum_{k=1}^{p}\left(c_{k}\right)_{1}\left(x_{1 r}^{\alpha}\left(t_{k}\right)\right)\right)+\int_{0}^{T}(3-\alpha) S_{1 r}^{\alpha}(T-s) s\left(x_{1 r}^{\alpha}(s)\right)^{2} d s\right]\right), } \\
u_{2 l}^{\alpha}(s)=\left(\tilde{\beta}_{2 l}^{\alpha}\right)^{-1}( & (\alpha+2)-\sum_{k=1}^{p}\left(c_{k}\right)_{2}\left(x_{i l}^{\alpha}\left(t_{k}\right)\right) \\
- & {\left.\left[S_{2 l}^{\alpha}(T)\left(\left(x_{0}\right)_{2 l}^{\alpha}-\sum_{k=1}^{p}\left(c_{k}\right)_{2}\left(x_{2 l}^{\alpha}\left(t_{k}\right)\right)\right)+\int_{0}^{T}(\alpha+1) S_{2 l}^{\alpha}(T-s) s\left(x_{2 l}^{\alpha}(s)\right)^{2} d s\right]\right), } \\
- & {\left.\left[S_{2 r}^{\alpha}(T)\left(\left(x_{0}\right)_{2 r}^{\alpha}-\sum_{k=1}^{p}\left(c_{k}\right)\left(x_{2 r}^{\alpha}\left(t_{k}\right)\right)\right)+\int_{0}^{T}(3-\alpha) S_{2 r}^{\alpha}(T-s) s\left(x_{2 r}^{\alpha}(s)\right)^{2} d s\right]\right) . } \\
u_{2 r}^{\alpha}(s)=\left(\tilde{\beta}_{2 r}^{\alpha}\right)^{-1}( & (4-\alpha)-\sum_{k=1}^{p}\left(c_{k}\right)_{2}\left(x_{i r}^{\alpha}\left(t_{k}\right)\right) \\
&
\end{aligned}
$$


Then $\alpha$-level of $x(T)=\left(x_{1}(T), x_{2}(T)\right)$ is

$$
\begin{aligned}
& {\left[x_{1}(T)\right]^{\alpha}} \\
& =\left[x_{1 l}^{\alpha}(T), x_{1 r}^{\alpha}(T)\right] \\
& =\left[S_{1 l}^{\alpha}(T)\left(\left(x_{0}\right)_{1 l}^{\alpha}-\sum_{k=1}^{p}\left(c_{k}\right)_{1}\left(x_{1 l}^{\alpha}\left(t_{k}\right)\right)\right)+\int_{0}^{T}(\alpha+1) S_{1 l}^{\alpha}(T-s) s\left(x_{1 l}^{\alpha}(s)\right)^{2} d s\right. \\
& \quad+\tilde{\beta}_{1 l}^{\alpha}\left(\tilde{\beta}_{1 l}^{\alpha}\right)^{-1}\left((\alpha+1)-\sum_{k=1}^{p}\left(c_{k}\right)_{1}\left(x_{i l}^{\alpha}\left(t_{k}\right)\right)\right. \\
& \quad-\left\{S_{1 l}^{\alpha}(T)\left(\left(x_{0}\right)_{1 l}^{\alpha}-\sum_{k=1}^{p}\left(c_{k}\right)_{1}\left(x_{1 l}^{\alpha}\left(t_{k}\right)\right)\right)\right. \\
& \left.\left.\quad+\int_{0}^{T}(\alpha+1) S_{1 l}^{\alpha}(T-s) s\left(x_{1 l}^{\alpha}(s)\right)^{2} d s\right\}\right) d s \\
& \quad S_{1 r}^{\alpha}(T)\left(\left(x_{0}\right)_{1 r}^{\alpha}-\sum_{k=1}^{p}\left(c_{k}\right)_{1}\left(x_{1 r}^{\alpha}\left(t_{k}\right)\right)\right)+\int_{0}^{T}(3-\alpha) S_{1 r}^{\alpha}(T-s) s\left(x_{1 r}^{\alpha}(s)\right)^{2} d s \\
& \quad+\tilde{\beta}_{1 r}^{\alpha}\left(\tilde{\beta}_{1 r}^{\alpha}\right)^{-1}\left((3-\alpha)-\sum_{k=1}^{p}\left(c_{k}\right)_{1}\left(x_{1 r}^{\alpha}\left(t_{k}\right)\right)\right. \\
& \quad+\int_{1 r}^{\alpha}(T)\left(\left(x_{0}\right)_{1 r}^{\alpha}-\sum_{k=1}^{p}\left(c_{k}\right)_{1}\left(x_{1 r}^{\alpha}\left(t_{k}\right)\right)\right) \\
& \left.\left.(\alpha+1)-\sum_{k=1}^{p}\left(c_{k}\right)_{1}\left(x_{1 l}^{\alpha}\left(t_{k}\right)\right),(3-\alpha)-\sum_{k=1}^{p}\left(c_{k}\right)_{1}\left(x_{1 r}^{\alpha}\left(t_{k}\right)\right)\right]=\left[\tilde{2}-\sum_{k=1}^{p}\left(c_{k}\right)_{1}\left(x_{1}\left(t_{k}\right)\right)\right]^{\alpha}\right)
\end{aligned}
$$

Similarly

$$
\left[x_{2}(T)\right]^{\alpha}=\left[x_{2 l}^{\alpha}(T), x_{2 r}^{\alpha}(T)\right]=\left[\widetilde{3}-\sum_{k=1}^{p}\left(c_{k}\right)_{2}\left(x_{2}\left(t_{k}\right)\right)\right]^{\alpha} .
$$

Hence

$$
\begin{aligned}
x(T) & =\left(x_{1}(T), x_{2}(T)\right) \\
& =\left(\tilde{2}-\sum_{k=1}^{p}\left(c_{k}\right)_{1}\left(x_{1}\left(t_{k}\right)\right), \tilde{3}-\sum_{k=1}^{p}\left(c_{k}\right)_{2}\left(x_{2}\left(t_{k}\right)\right)\right)=x^{1}-g(x) .
\end{aligned}
$$

Then all the conditions stated in Theorem 4.2 are satisfied, so system (5.2) is nonlocal controllable on $[0, T]$. 


\section{Acknowledgment}

This study was supported by research funds from Dong-A University.

\section{References}

[1] P. Diamond and P. Kloeden, Metric Spaces of Fuzzy Sets: Theory and Applications, World Scientific, River Edge, NJ, USA, 1994.

[2] Y. C. Kwun and D. G. Park, “Optimal control problem for fuzzy differential equations,” in Proceedings of the Korea-Vietnam Joint Seminar, pp. 103-114, 1998.

[3] P. Balasubramaniam and S. Muralisankar, "Existence and uniqueness of fuzzy solution for semilinear fuzzy integrodifferential equations with nonlocal conditions," Computers \& Mathematics with Applications, vol. 47, no. 6-7, pp. 1115-1122, 2004.

[4] J. H. Park, J. S. Park, and Y. C. Kwun, “Controllability for the semilinear fuzzy integrodifferential equations with nonlocal conditions," in Fuzzy Systems and Knowledge Discovery, vol. 4223 of Lecture Notes in Computer Science, pp. 221-230, Springer, Berlin, Germany.

[5] Y. C. Kwun, M. J. Kim, B. Y. Lee, and J. H. Park, "Existence of solutions for the semilinear fuzzy integrodifferential equations using by succesive iteration," Journal of Korean Institute of Intelligent Systems, vol. 18, pp. 543-548, 2008.

[6] Y. C. Kwun, M. J. Kim, J. S. Park, and J. H. Park, "Continuously initial observability for the semilinear fuzzy integrodifferential equations," in Proceedings of the 5th International Conference on Fuzzy Systems and Knowledge Discovery, vol. 1, pp. 225-229, Jinan, China, October 2008.

[7] B. Bede and S. G. Gal, "Almost periodic fuzzy-number-valued functions," Fuzzy Sets and Systems, vol. 147, no. 3, pp. 385-403, 2004.

[8] S. G. Gal and G. M. N'Guérékata, "Almost automorphic fuzzy-number-valued functions," Journal of Fuzzy Mathematics, vol. 13, no. 1, pp. 185-208, 2005.

[9] G. Wang, Y. Li, and C. Wen, “On fuzzy $n$-cell numbers and $n$-dimension fuzzy vectors," Fuzzy Sets and Systems, vol. 158, no. 1, pp. 71-84, 2007. 